\title{
Rotating Flows Driven by Axisymmetric Blades in a Cylinder*
}

\author{
JAE WON KIM \\ Department of Mechanical Engineering, Sun Moon University, Asan City, Chung Nam 336-840, South Korea
}

(Received 24 April 1998; Revised 8 May 1998; In final form 7 July 1998)

\begin{abstract}
A study is made of steady state flow of a viscous fluid, driven by a rotating endwall disk having radial blades in a finite geometry. Numerical solutions to the Navier-Stokes equations are obtained for the flows in the cylindrical cavity. The bottom endwall disk of the vessel is impulsively rotating at a constant rotating speed $\Omega$ with respect to the longitudinal axis of it. Details of the three components velocity field are examined at high Reynolds number for its engineering application. The main parameter for this study is the number of the radial blades of a rotating pulsator. The numerical results for the fluid flows and pressure distribution, for both an odd and an even number of the blades are procured. The present output offers an optimal number of the blades for rotating machinery such as agitator. The grid-net for the numerical computation is constructed on a body-fitted coordinate system transformed from physical coordinates. It is also flexible to suit any number of blades attached on the rotating bottom disk. The algorithm for the numerical computation is based on the SIMPLE release by Patankar, and the results are validated with prior published data. In addition, a characteristic model is prepared for the pressure measurement. The pressure measurements performed for the present model are consistent with this computational work. The explicit effect of the blade on the overall flow character is scrutinized. The numerical data are processed to describe the behavior of the meridional velocities under different blade conditions. Also, the traces of particles are plotted to assess the effects. Pronounced differences are noted and these results supply comprehensive data for practical application.
\end{abstract}

Keywords: Rotating flow, Rotating blade, Meridional flow, Numerical work, Body-fitted coordinate, Computational work

\section{INTRODUCTION}

Fluid flows in a circular cylinder with a rotating exciter which is, in fact, radial blades on its surface has been studied in this paper. The present model describes the characteristics of the flow system in an upright washing machine. The characteristic model consist of a closed circular cavity fully filled with viscous fluid of kinematic viscosity $\nu$, and its endwall disks. The top disk of the cylinder is

* This paper was originally presented at ISROMAC-7.

${ }^{\dagger}$ Tel.: +824185302337. Fax: +824185420141. E-mail: jwk@omega.sunmoon.ac.kr. 
stationary, whereas the rotating bottom one causes fluid motion inside the vessel of radius $R$. The aspect ratio of the container is 2.5. Figure 1 schematically shows the flow system.

Rotating flows in a circular cylinder with flat endwall disks have been extensively investigated in recent years (Larrousse, 1987; Altgeld et al., 1983; Escudier, 1984). This internal flow serves as a classical model, exhibiting the dynamic ingredients intrinsic to rotating fluid systems. Also, the flows confined between two parallel coaxial disks, one rotating and the other stationary, are of great relevance to a host of technological applications, i.e., fluid machinery, chemical mixers, centrifuges, computer disk drive systems, and washing machine systems, to name a few. For a definitive problem formulation, it will be envisioned that the cylinder axis is aligned vertically and the spinning disk forms the bottom endwall of the cylinder. One principal dynamic component is the Ekman layer that forms on the endwall disks perpendicular to the rotation

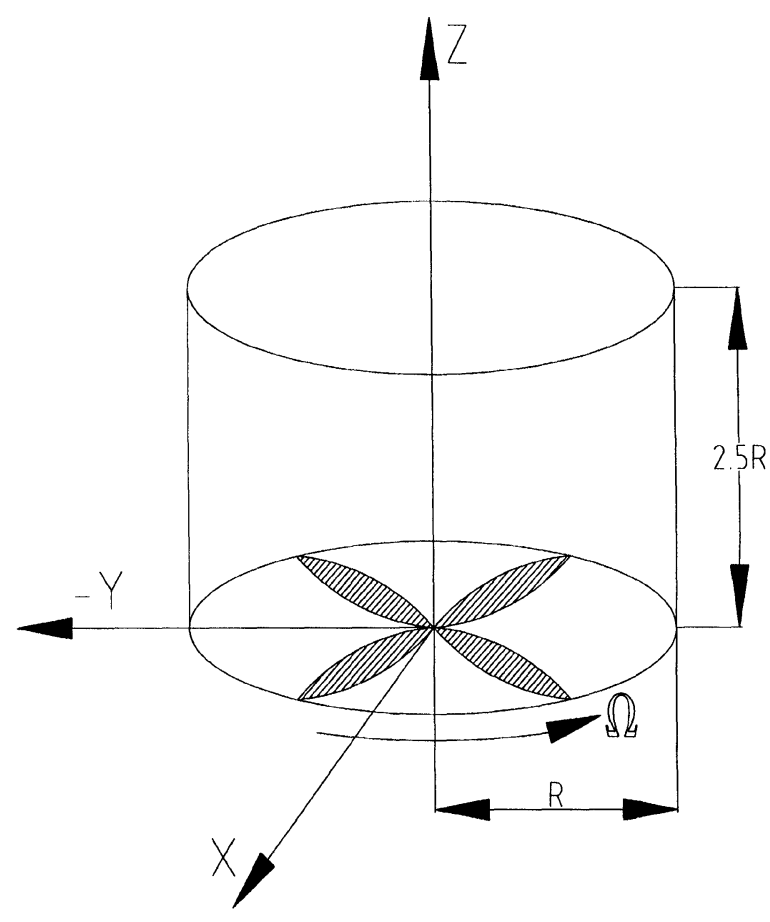

FIGURE 1 Schematic diagram of flow system and coordinates. axis. Owing to the fluid pumping mechanism by the Ekman layer, a small axial flow is induced; this in turn gives rise to the secondary flows in the meridional plane. In practice, the basic idea for this type washing machine with a rotating pulsator at the bottom of the tub is to utilize the secondary flows in a meridional plane of a cavity as major mass transfer mechanism. The prior works are mainly on the flows system by a flat plate disk not a topological disk. Engineering data from the previous results are concerned with the flows by a rotating flat disk. In fact the difference between the rotating flows by a flat disk and bumpy disk is more pronounced as the rotational Reynolds number $\left(\operatorname{Re} \equiv \Omega R^{2} / \nu\right)$, based on radius of the cavity, increases. In engineering applications, Reynolds number is sufficiently large. Consequently, the present consideration is focused on the dramatic flows by a projecting part, i.e., a rotating convex blades attached on the endwall disk.

This investigation tries to find out the optimal number of blades for the rotating pulsator of the washer used by Asian customers. The number of blades of a pulsator in the present running model of washing machine in a market is equal to 6 . In this work, the estimation of the normal system and comparison between odd and even number of blades are both made for engineering applications. Both proper numerical scheme and grid system was prepared for this investigation. For the curved shape of the blades, the present mesh is non-orthogonal system and governing equations are also transformed into the body-fitted coordinate system. In detail, the blade is made of both typical sine curves and a horizontal line connecting each end points of the curved line. The flows in a meridional plane and spiral motion are described for several system with different number of vanes.

\section{NUMERICAL MODEL}

A schematic of the flow configuration, together with the physical coordinate system $(x, y, z)$ is shown in Fig. 1. For $t<0$, both the cylinder and 
the fluid are at rest. At the initial instant $t=0$, the bottom disk with blades begins impulsively rotating about the longitudinal axis $z$ with a constant speed, $\Omega$. As for usual engineering applications, the situation in which the rotational Reynolds number is large shall be considered. The task is to describe the induced flow motion in a tub by the topological rotating plate.

This numerical work is composed of two steps; first work is to run a program for numerical grid generation and then a solver program integrates each derivative term of governing equations. The grid system is constructed of four parts. Figure 2 displays the four groups, i.e., the disk with convex vanes, the vertical grid wall through the meridional plane defined by the two endwall disks and shrouded wall, the top disk, and the side wall of the cylindrical container. The present grid generation of the pulsator is so flexible that arbitrary number of blades is represented on the flat disk. In Fig. 2, typical pulsator with three radial blades on the flat plate bottom disk is constructed at the lower endwall disk.

The governing equations are the three dimensional incompressible Navier-Stokes equations (Eq. (1)) and continuity equation (Eq. (2)). Numerical calculation was carried out on the computational domain in the transformed coordinates $(\xi, \eta, \zeta)$ from the physical one $(x, y, z)$. Written for computational coordinates $(\xi, \eta, \zeta)$ with respective
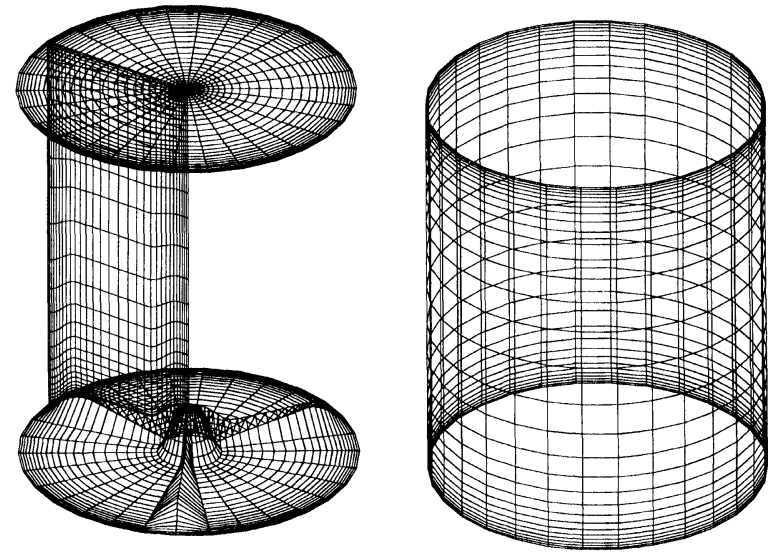

FIGURE 2 Grid net for flow configuration. non-dimensional velocity components $(U, V, W)$ these are

$$
\begin{aligned}
& \frac{1}{J}\left[\frac{\partial}{\partial \xi}(U \phi)+\frac{\partial}{\partial \eta}(V \phi)+\frac{\partial}{\partial \zeta}(W \phi)\right] \\
& =\frac{1}{J}\left\{\frac{\partial}{\partial \xi}\left[\Gamma\left(G^{11} \frac{\partial \phi}{\partial \xi}+G^{12} \frac{\partial \phi}{\partial \eta}+G^{13} \frac{\partial \phi}{\partial \zeta}\right)\right]\right\} \\
& +\frac{1}{J}\left\{\frac{\partial}{\partial \eta}\left[\Gamma\left(G^{21} \frac{\partial \phi}{\partial \xi}+G^{22} \frac{\partial \phi}{\partial \eta}+G^{23} \frac{\partial \phi}{\partial \zeta}\right)\right]\right\} \\
& +\frac{1}{J}\left\{\frac{\partial}{\partial \zeta}\left[\Gamma\left(G^{31} \frac{\partial \phi}{\partial \xi}+G^{32} \frac{\partial \phi}{\partial \eta}+G^{33} \frac{\partial \phi}{\partial \zeta}\right)\right]\right\} \\
& +S_{\phi}(\xi, \eta, \zeta) \text {. } \\
& \frac{1}{J}\left[\frac{\partial}{\partial \xi}(U)+\frac{\partial}{\partial \eta}(V)+\frac{\partial}{\partial \zeta}(W)\right]=0 . \\
& J=x_{\xi} y_{\eta} z_{\zeta}+x_{\zeta} y_{\xi} z_{\eta}+x_{\eta} y_{\zeta} z_{\xi}-x_{\xi} y_{\zeta} z_{\eta} \\
& -x_{\zeta} y_{\eta} z_{\xi}-x_{\eta} y_{\xi} z_{\zeta} \text {, } \\
& \xi_{x}=\frac{y_{\eta} z_{\zeta}-y_{\zeta} z_{\eta}}{J}, \quad \xi_{y}=\frac{x_{\zeta} z_{\eta}-x_{\eta} z_{\zeta}}{J}, \\
& \xi_{z}=\frac{x_{\eta} y_{\zeta}-x_{\zeta} y_{\eta}}{J}, \\
& \eta_{x}=\frac{y_{\zeta} z_{\xi}-y_{\xi} z_{\zeta}}{J}, \quad \eta_{y}=\frac{x_{\xi} z_{\zeta}-x_{\zeta} z_{\xi}}{J}, \\
& \eta_{z}=\frac{x_{\zeta} y_{\xi}-x_{\xi} y_{\zeta}}{J}, \\
& \zeta_{x}=\frac{y_{\xi} z_{\eta}-y_{\eta} z_{\xi}}{J}, \quad \zeta_{y}=\frac{x_{\eta} z_{\xi}-x_{\xi} z_{\eta}}{J}, \\
& \zeta_{z}=\frac{x_{\xi} y_{\eta}-x_{\eta} y_{\xi}}{J}, \\
& G^{11}=J\left(\xi_{x}^{2}+\xi_{y}^{2}+\xi_{z}^{2}\right), \\
& G^{31}=G^{13}=J\left(\zeta_{x} \xi_{x}+\zeta_{y} \xi_{y}+\zeta_{z} \xi_{z}\right), \\
& G^{23}=G^{32}=J\left(\eta_{x} \zeta_{x}+\eta_{y} \zeta_{y}+\eta_{z} \zeta_{z}\right), \\
& G^{12}=G^{21}=J\left(\xi_{x} \eta_{x}+\xi_{y} \eta_{y}+\xi_{z} \eta_{z}\right), \\
& G^{33}=J\left(\zeta_{x}^{2}+\zeta_{y}^{2}+\zeta_{z}^{2}\right), \\
& G^{22}=J\left(\eta_{x}^{2}+\eta_{y}^{2}+\eta_{z}^{2}\right) .
\end{aligned}
$$

Consider a vertically mounted right circular cylinder of radius $R$ and height $2.5 R$ filled with a fluid having kinematic viscosity $\nu$. The aspect ratio of the present cavity, $A$, is equal to 2.5 in this numerical work. The rotating bottom disk with 
convex radial vanes cause internal flows of a vessel. The bottom disk rotates steadily about the central axis at constant angular frequency $\Omega$. The characteristic variables for normalization are $R, R \Omega$, and $1 / \Omega$ for length, velocity, and time, respectively.

The actual computational procedure is as following. At the absolutely silent flow, the impulsively rotation of the uneven bottom disk is imposed in the flow field.

The main focus is on the flow field at almost steady state. In summary, the initial and boundary conditions can be expressed as

$$
U=V=W=0 \text { for } t \leq 0 \quad \text { (initial state) }
$$

$$
U=V=W=0 \quad \text { at } r=R \text { for } 0<z<2.5 R,
$$$$
t>0 \quad \text { (the side wall), }
$$

$U=V=W=0 \quad$ at $z=2.5 R$ for $0<r<R$,

$$
t>0 \text { (the top disk), }
$$

$V=r / R \quad$ at $z=0$ for $0<r<R$,

$$
t>0 \text { (the bottom disk). }
$$

The time-marching integration continued until an approximate steady state was attained. The spin-up time scale, $\tau=\operatorname{Re}^{1 / 2} \Omega^{-1}$, gauged the lapse of time for global adjustment in unsteady rotating flows (Hyun and Kim, 1989; Kim and Hyun, 1997). For convenience, the approximate steady state in the present work was arbitrarily taken to be the state when the flow variables at $r / R=0.5, z / R=1.25$, changed less than $0.1 \%$ over the time interval $0.1 \tau$.

The finite-volume methods developed by Patankar (1980) were amended to solve the system of equations. This method has been shown to provide accurate and reliable numerical solutions to a large class of rotating fluid flows in the manuscripts by Kim and Noh (1997) and Lang et al. (1994). This model used the primitive variables on a regular nonuniform mesh. The pressure was found from a Poisson equation, which was solved by a pressure substitution method proposed by Hobson and Lakshminarayana (1991). The specifics of the numerical techniques adopted, including the finite-volume method, were elaborated in Kim and Noh (1997). Also this numerical result obtains its accuracy by validation both with the prior work and comparison with laboratory experiments of pressure in a model system.

In order to validate the present numerical results comprehensive experiment has been performed Pressure variation in the flow system is measured and compared with the numerical data. Figure 3 shows the axial profiles of pressure at a radial location, $r / R=0.75$. The continuous line denotes the numerical results and the symbols for the measurements by a pressure transducer that is connected to a hole via a fitting tube. The holes for the pressure gauging are prepared on a rod mounted at a wanted position. Uncertainty error due to the rod and tube for pressure transfer is rigourously excluded by removing bubbles in the experiment system. It is observed that the discrepancy between the two data is in a reasonable range.

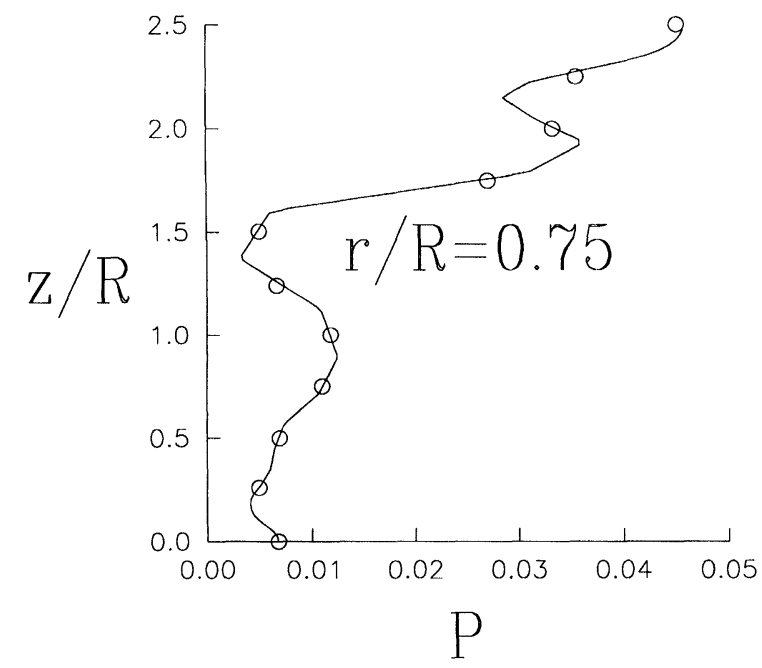

FIGURE 3 Comparison of pressure by computational work (solid line) and measurements (symbols) along axial locations at $r / R=0.75$. 


\section{RESULTS AND DISCUSSION}

Several sets of numerical computations were conducted to render comprehensive flow details. For all the calculations performed, $\operatorname{Re}=2 \times 10^{3}$ and $A=2.5$. These values are comparable to those used in the real running model. In line with the stated objectives of the present study, the number blades were varied to scrutinize the effects of suction in finite geometry. In the ensuring discussion, only the exemplary and physical illuminating results will be presented.

Figure 4 displays the vector plot of the meridional velocities in a vertical cross plane $(x=0$ plane) according to the number of blades. Figure 4(a) shows the plot for the case of $B=2$, where $B$ denotes the number of blade on the rotating disk. Also the flows for $B=3,5$ and 6 are depicted in Fig. 4(b)-(d), respectively. The cross sectional plane for this plot is the vertical meridional one constituted by $x$ - and $z$-axis. The flows are asymmetric for the case of odd number blades. Otherwise symmetric flow is found for even number blades (see Fig. 4(d) for $B=6$ ).

Similar plot in the $y=0$ plane for $B=3$ is displayed in Fig. 5. Flows are still asymmetric and only right half of the wing is shown in the plane.

In the plot of meridional velocities, it is observed that there are secondary circulations due to the action of suction by a rotating pulsator. Major emphasis is on the enhanced meridional circulation by adjustment of the number of wings. In this work, the magnitude of the meridional velocity is indirectly gauged by the quantity of the axial velocity at a pre-fixed depth of fluid in $x=0$ plane. For convenience, the depth of water for the measurement is at $z / R=0.5$. Figure 6 shows the radial variation of the axial velocity in $x=0$ plane depending on the number of wings. The meridional flow is axisymmetric with respect to the vertical axis in the case of even number of $B$.

In contrast, the flows are not axisymmetric for odd number of $B$. For the present rotational condition, in the case of $R e=2000$, it is found that the downward flows toward the bottom disk for
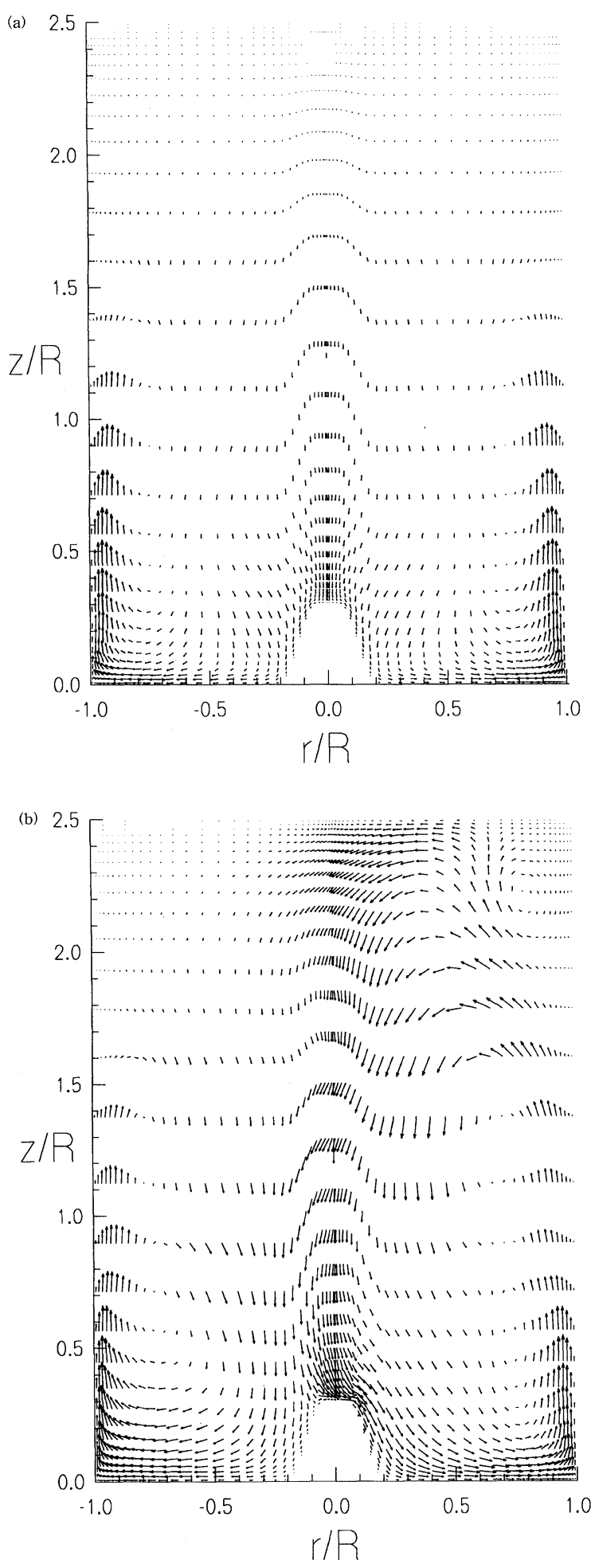

FIGURE 4(a) and (b) 

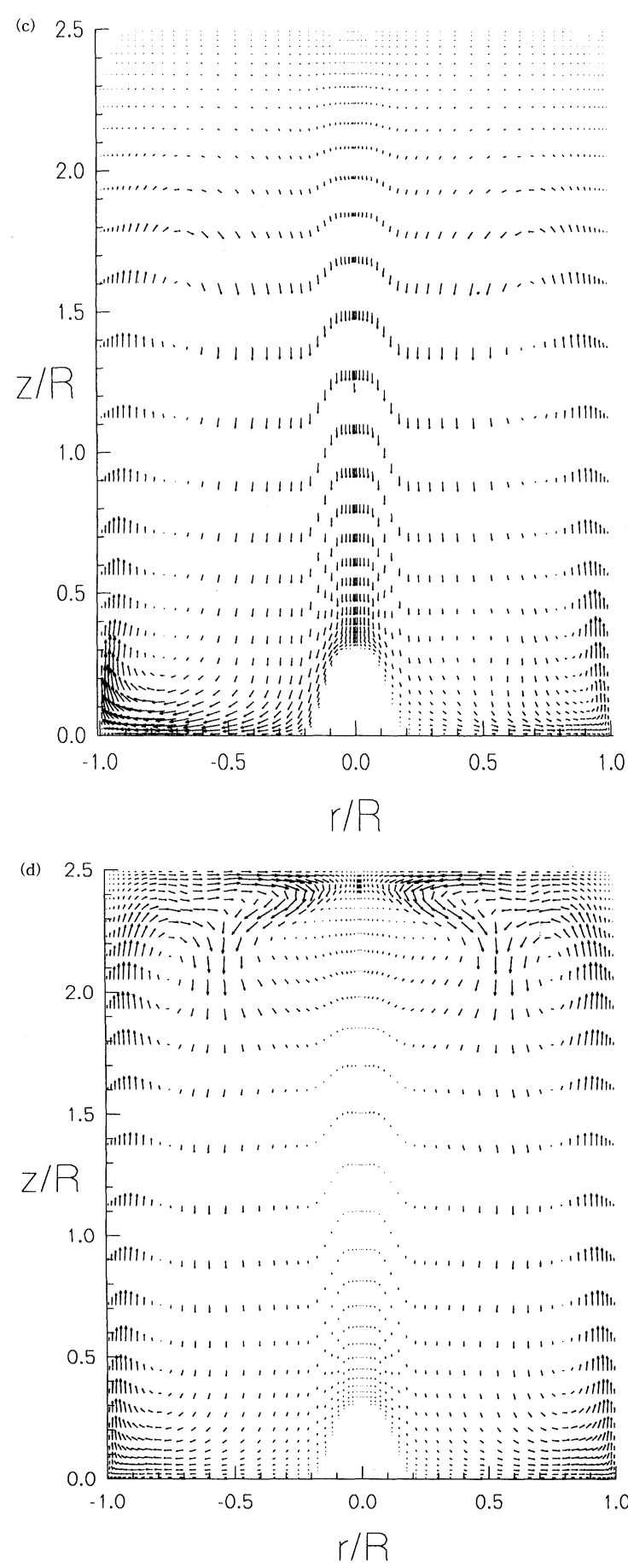

FIGURE 4(c) and (d)

FIGURE 4 Meridional velocity vector plot in the $x=0$ plane according to (a) $B=2$, (b) $B=3$, (c) $B=5$, and (d) $B=6$.

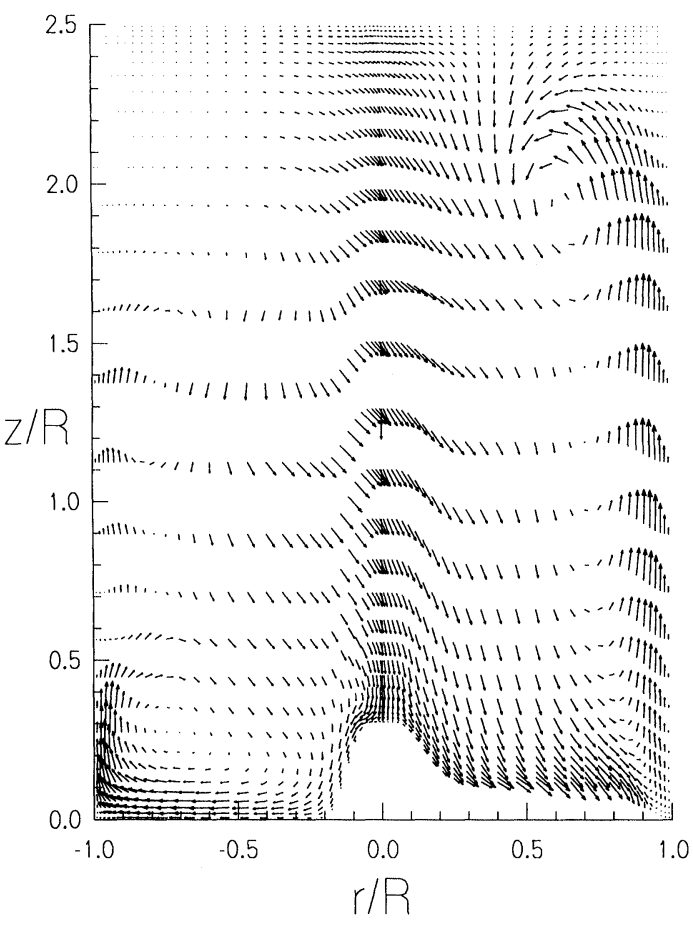

FIGURE 5 Vector plot for $B=3$ in the $y=0$ plane.

both cases of $B=5$ and 6 are obstructed and restricted because of excess vanes. The analysis of Fig. 6 shows the optimal number of blades for $\mathrm{Re}=2000$ is less than 5 .

Let us compare the suction velocity in the another meridional plane. Figure 7 shows the comparison of the magnitude of the vertical velocity according to the meridional planes for same blade number, $B=3$. Figure 7(a) displays the radial variation of the vertical velocity $(W)$ in $x=0$ plane and 7(b) in $y=0$ plane which is cross sectional plane constructed by $x$-axis and the vertical axis. In the two plots, the magnitude and shape are changed depending on the view-plane. However, the characteristics of asymmetry are still found in the both two plots.

As stated earlier, the resulting flows are symmetric or asymmetric depending on the number of blades. It is recommended for the enhanced radial flows to select the odd number of wings because the asymmetric behavior of the meridional flows cause the inward and outward flows along the vertical axis. 

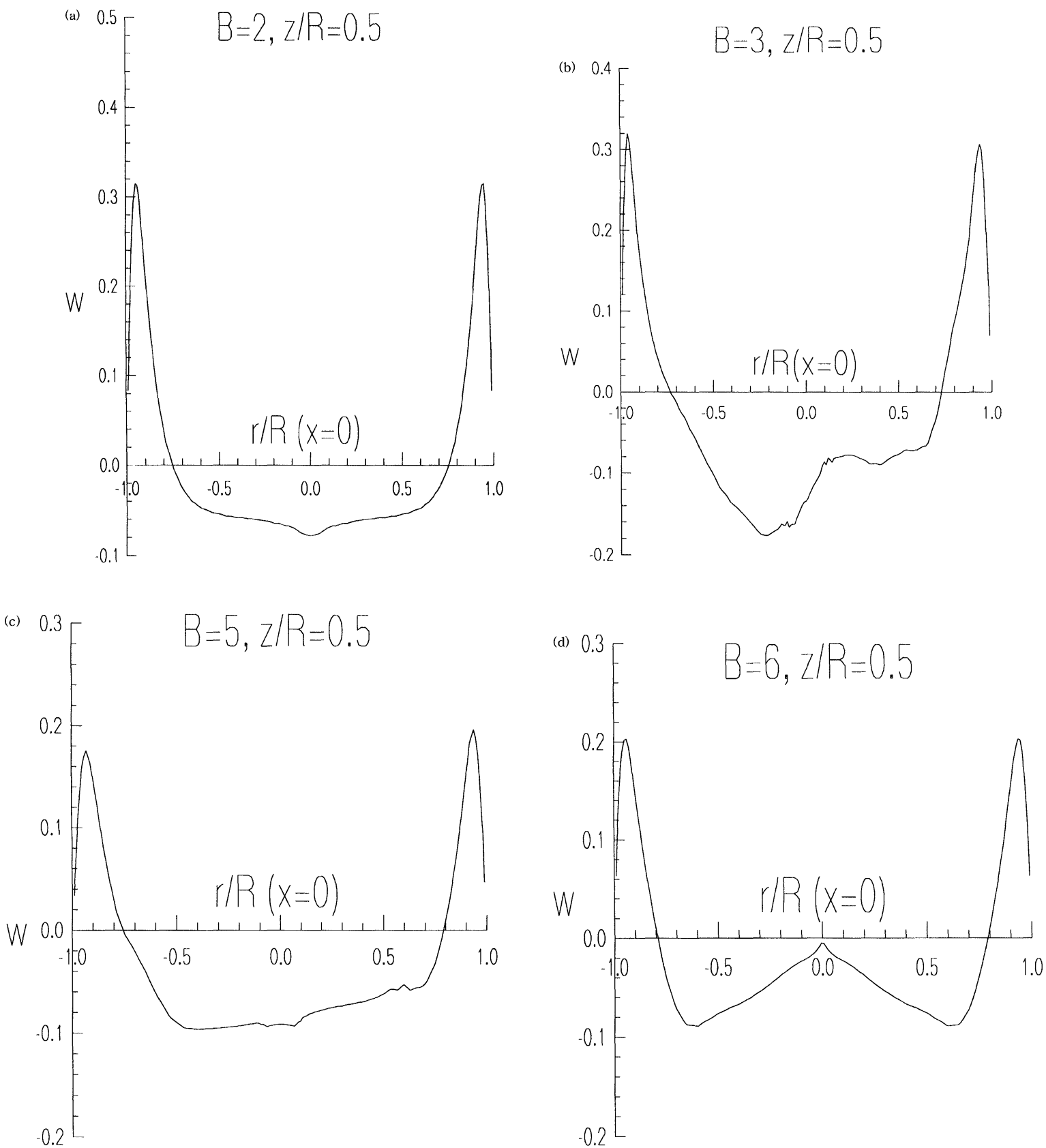

FIGURE 6 Radial variation of the axial velocity $(W)$ near the bottom disk in the $x=0$ plane. (a) $B=2,($ b) $B=3$, (c) $B=5$, and (d) $B=6$.

\section{REMARKS}

Numerical calculation for the flow filed in a model of a washing tub having a pulsator is carried out for the selection of the number of blades of a rotating agitator. The present grid system represents the overall domain of the flow configuration involving the uneven shape of a pulsator. The result 
(a)

$B=3, z / R=0.5$

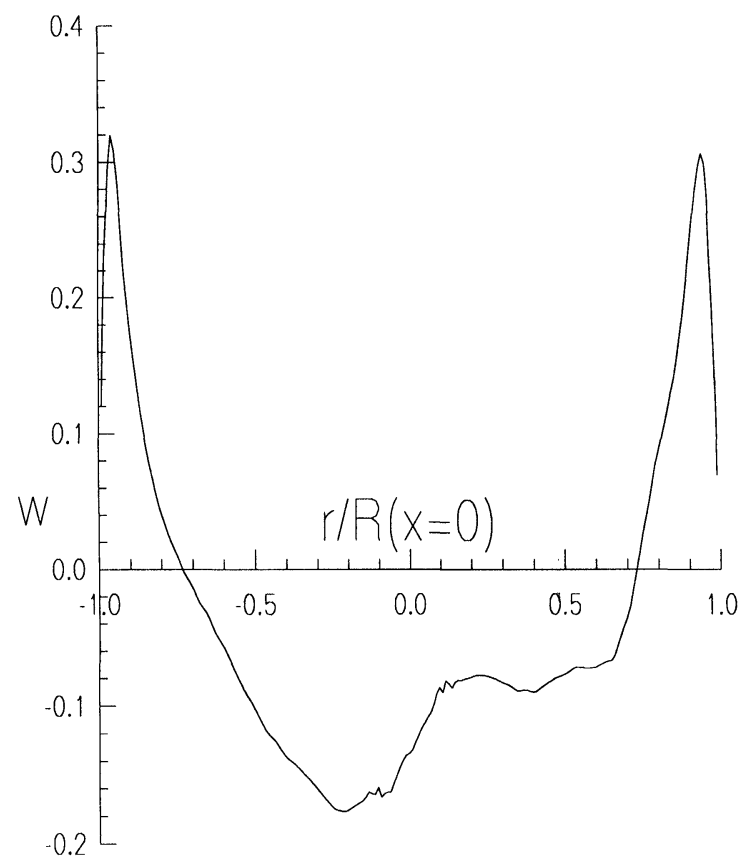

(b)

$B=3,2 / R=0.5$

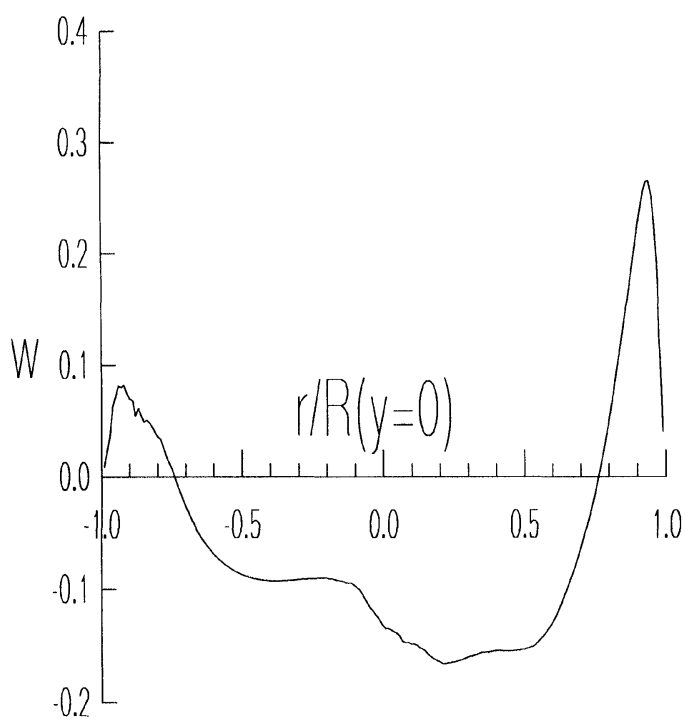

FIGURE 7 Plots of vertical velocity in the $x=0$ plane (a) and $y=0$ plane (b).

of the present numerical calculations is compared with the advanced work by Lang et al. (1994) and is in good agreement. Also the solution by this numerical work is directly validated by laboratory experiments.

For this rotating flows at $\mathrm{Re}=2000$, the optimal number of wings of the rotating pulsator is 3 for odd number and 4 for even number. The meridional flows for mixing mechanism when the number of wings is five or six becomes weaker and weaker due to the inconsistency between the time interval of the rotating blade and the phase of downward velocity by the suction of the rotating endwall. The suction speed by the pulsator with 3 or 4 blades is faster than that induced by the rotating plate having 5 or 6 blades. The role of the vane of a pulsator is defined as a generator of suction motion in this research.

Meridional flows are axisymmetric for even number of risen vanes from the bottom disk. In the case of asymmetric flow, the blade number is odd. The difference of odd and even blade numbers is the symmetric or asymmetric behavior of the flows by the pulsator.

\section{Acknowledgment}

This work was supported by the Korea Science and Engineering Foundation through the Regional Research Center for Climate Control Technology at Sun Moon University, Korea.

\section{NOMENCLATURE}

$\begin{array}{ll}A & \text { aspect ratio of the cavity } \\ B & \text { number of blades } \\ J & \text { Jacobian matrix } \\ p & \text { pressure normalized by } \rho \Omega R^{2} \\ R & \text { radius of cylinder }\end{array}$


$\operatorname{Re}$

$t$

$(U, V, W)$

$(x, y, z)$

$(\xi, \eta, \zeta)$

$\rho$

$\nu$

$\phi$

$\Omega$

\section{References}

Altgeld, H., Jones, W.P. and Wilhelmi, H., 1983, Velocity measurements in a confined swirl driven recirculating flow, Experiments in Fluids, 1, 236-242.
Escudier, M.P., 1984, Observations of the flow produced in a cylindrical container by a rotating endwall, Experiments in Fluids, 2, 189-196.

Hobson, G.V. and Lakshminarayana, B., 1991, Prediction of cascade performance using an incompressible Navier-Stokes technique, ASME Journal of Turbomachinery, 113, 561-572.

Hyun, J.M. and Kim, J.W., 1989, Buoyant convection driven by an encapsuled spinning disk with axial suction, AIAA $J$. Thermophysics and Heat Transfer, 3(2), 189-194.

Kim, J.W. and Hyun, J.M.. 1997, Axisymmetric inertial oscillations in transient rotating flows in a cylinder, ASME J. Fluids Engineering, 119(2), 390-396.

Kim, J.W. and Noh, S.K., 1997, Enhancement of mixing effects in fluid flows having an inclined endwall, Proceedings of ExHFT-4, Brussels, Vol. III, pp. 1505-1510.

Lang, E., Sridhar, K. and Wilson, N.M., 1994, Computational study of disk driven rotating flow in a cylindrical enclosure, ASME J. Fluids Engineering, 116(4), 621-629.

Larrousse, M.F., 1987, Transport Phenomena During Spin-up/ Spin-down in the Bridgman-Stokbarger Technique, Clarkson University, New York.

Patankar, S.V., 1980, Numerical Heat Transfer and Fluid Flow, Hemisphere Publishing Company, New York. 


\section{ait \\ ENERGY MATERIALS}

M A N E Y publishing

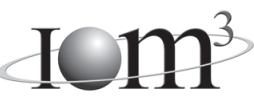

\section{Materials Science \& Engineering for Energy Systems}

Maney Publishing on behalf of the Institute of Materials, Minerals and Mining

The Institute of Materials, Minerals \& Mining

Economic and environmental factors are creating ever greater pressures for the efficient generation, transmission and use of energy. Materials developments are crucial to progress in all these areas: to innovation in design; to extending lifetime and maintenance intervals; and to successful operation in more demanding environments. Drawing together the broad community with interests in these areas, Energy Materials addresses materials needs in future energy generation, transmission, utilisation, conservation and storage. The journal covers thermal generation and gas turbines; renewable power (wind, wave, tidal, hydro, solar and geothermal); fuel cells (low and high temperature); materials issues relevant to biomass and biotechnology; nuclear power generation (fission and fusion); hydrogen generation and storage in the context of the 'hydrogen economy'; and the transmission and storage of the energy produced.

As well as publishing high-quality peer-reviewed research, Energy Materials promotes discussion of issues common to all sectors, through commissioned reviews and commentaries. The journal includes coverage of energy economics and policy, and broader social issues, since the political and legislative context influence research and investment decisions.

\section{CALL FOR PAPERS}

Contributions to the journal should be submitted online at http://ema.edmgr.com

To view the Notes for Contributors please visit: www.maney.co.uk/journals/notes/ema

Upon publication in 2006, this journal will be available via the Ingenta Connect journals service. To view free sample content online visit: www.ingentaconnect.com/content/maney

For further information please contact:

Maney Publishing UK

Tel: +44 (0)113 2497481 Fax: +44 (0)1132486983 Email: subscriptions@maney.co.uk

or

Maney Publishing North America

Tel (toll free): 8662975154 Fax: 6173546875 Email: maney@maneyusa.com

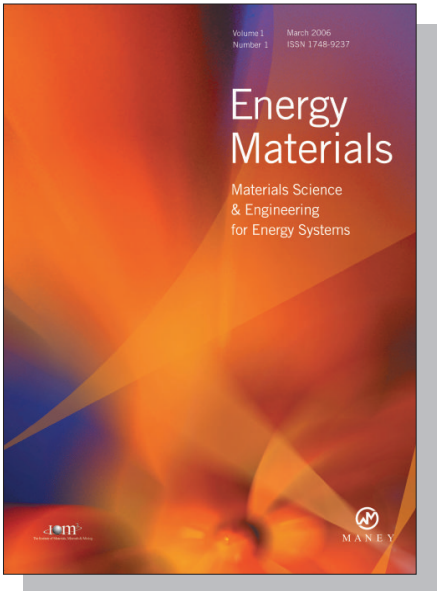

EDITORS

Dr Fujio Abe

NIMS, Japan

Dr John Hald, IPL-MPT, Technical University of Denmark, Denmark

Dr R Viswanathan, EPRI, USA

\section{SUBSCRIPTION INFORMATION}

Volume 1 (2006), 4 issues per year

Print ISSN: 1748-9237 Online ISSN: 1748-9245

Individual rate: $£ 76.00 / U S \$ 141.00$

Institutional rate: $£ 235.00 /$ US $\$ 435.00$

Online-only institutional rate: $£ 199.00 / U S \$ 367.00$

For special $\mathrm{IOM}^{3}$ member rates please email

subscriptions@maney.co.uk

\section{For further information or to subscribe online please visit www.maney.co.uk}



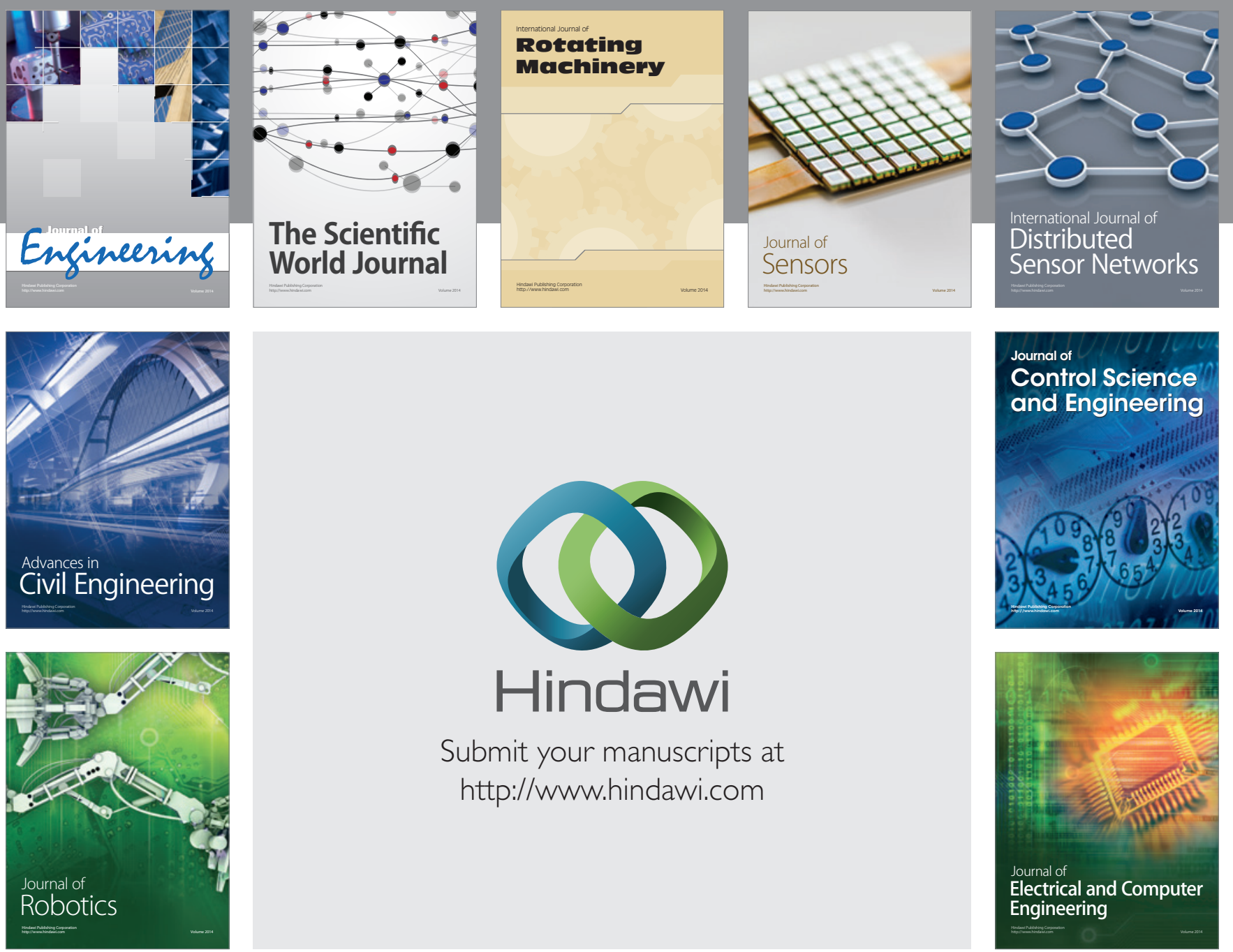

Submit your manuscripts at

http://www.hindawi.com
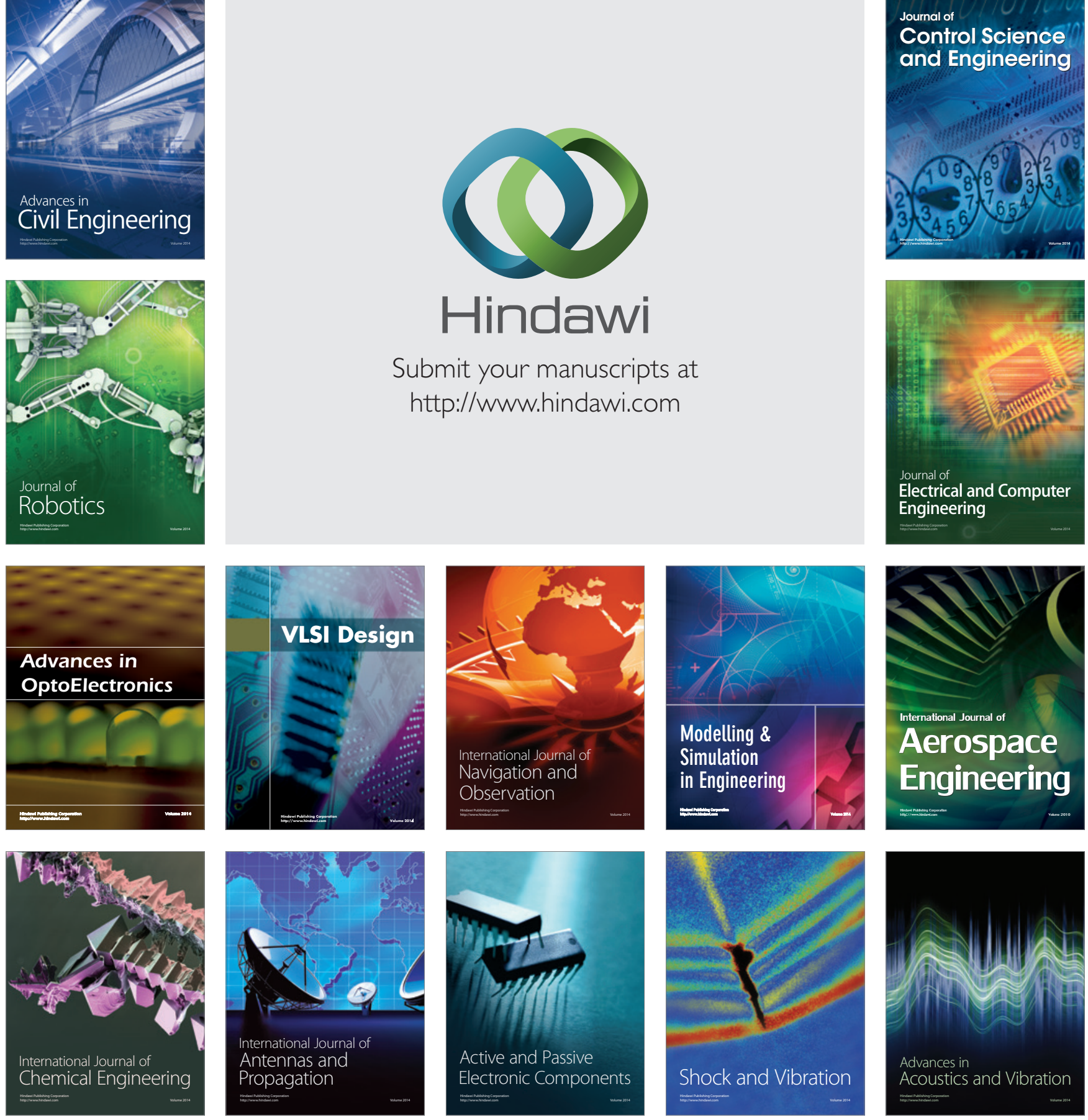